\title{
Measurement of the X-ray Spectrum of a Free Electron Laser with a Wide-Range High-Resolution Single-Shot Spectrometer
}

\author{
Yuichi Inubushi ${ }^{1,2, *}$, Ichiro Inoue ${ }^{2}$, Jangwoo Kim ${ }^{3,4}$, Akihiko Nishihara ${ }^{3}$, Satoshi Matsuyama ${ }^{3}$, \\ Hirokatsu Yumoto ${ }^{1,2}$, Takahisa Koyama ${ }^{1,2}$, Kensuke Tono ${ }^{1,2}$, Haruhiko Ohashi ${ }^{1,2}$, \\ Kazuto Yamauchi ${ }^{3}$ and Makina Yabashi ${ }^{1,2}$ \\ 1 Japan Synchrotron Radiation Research Institute, 1-1-1 Kouto, Sayo-cho, Sayo-gun, Hyogo 679-5198, Japan; \\ yumoto@spring8.or.jp (H.Y.); koyama@spring8.or.jp (T.K.); tono@spring8.or.jp (K.T.); \\ hohashi@spring8.or.jp (H.O.); yabashi@spring8.or.jp (M.Y.) \\ 2 RIKEN SPring-8 Center, 1-1-1 Kouto, Sayo-cho, Sayo-gun, Hyogo 679-5148, Japan; inoue@spring8.or.jp \\ 3 Department of Precision Science and Technology, Graduate School of Engineering, Osaka University, \\ 2-1 Yamada-oka, Suita, Osaka 565-0871, Japan; jkpal@postech.ac.kr (J.K.); \\ nishihara@up.prec.eng.osaka-u.ac.jp (A.N.); matsuyama@prec.eng.osaka-u.ac.jp (S.M.); \\ yamauchi@prec.eng.osaka-u.ac.jp (K.Y.) \\ 4 Pohang Accelerator Laboratory (PAL), POSTECH, 127-80 Jigokro, Nam-gu, Pohang, \\ Gyeongbuk 37673, Korea \\ * Correspondence: inubushi@spring8.or.jp; Tel.: +81-791-58-0802
}

Academic Editor: Kiyoshi Ueda

Received: 31 March 2017; Accepted: 30 May 2017; Published: 6 June 2017

\begin{abstract}
We developed a single-shot X-ray spectrometer for wide-range high-resolution measurements of Self-Amplified Spontaneous Emission (SASE) X-ray Free Electron Laser (XFEL) pulses. The spectrometer consists of a multi-layer elliptical mirror for producing a large divergence of $22 \mathrm{mrad}$ around $9070 \mathrm{eV}$ and a silicon (553) analyzer crystal. We achieved a wide energy range of $55 \mathrm{eV}$ with a fine spectral resolution of $80 \mathrm{meV}$, which enabled the observation of a whole SASE-XFEL spectrum with fully-resolved spike structures. We found that a SASE-XFEL pulse has around 60 longitudinal modes with a pulse duration of $7.7 \pm 1.1 \mathrm{fs}$.
\end{abstract}

Keywords: XFEL; spectroscopy; ultra-shot pulse

\section{Introduction}

Recently, X-ray Free Electron Lasers (XFELs), such as the Linac Coherent Light Source (LCLS) [1] and the SPring-8 Angstrom Compact Free Electron Laser (SACLA) [2], have successfully generated brilliant, femtosecond X-ray pulses, achieving an ultrahigh resolving power in both space and time that opens up new frontiers in various scientific fields [3-5]. Currently, the Self-Amplified Spontaneous Emission (SASE) scheme [6,7] is widely utilized for generating XFEL pulses. SASE-XFEL pulses have many narrow spike structures originating from the stochastic nature of the electron beam used as light source. The spike structures reflect important characteristics of the XFEL beam: the width and the number of spikes in the whole spectrum closely relate with the XFEL pulse duration and the longitudinal mode number, respectively. To measure spike structures, single-shot measurements with a dispersive X-ray spectrometer are required, because spike structures change shot by shot [8-11]. However, it was difficult to fully resolve the spike structure with a typical width of $\sim 100$ meV over the entire pulse spectrum covering a few tens of eV. In this paper, we report on the development of a single-shot spectrometer covering the whole XFEL spectrum with fully-resolved spike structures. 
So far, two methods have been developed for designing single-shot spectrometers of well-collimated XFEL beams. One uses a thin, bent analyzer crystal $[9,11]$. The total spectral range can be simply tuned by controlling the bending radius, while possible lattice distortions induced by the bending can degrade the energy resolution. The other one combines a flat analyzer crystal and an elliptical total-reflection mirror to increase the angular divergence of X-rays. In this case, one can achieve high resolution of $14 \mathrm{meV}$ at around $10 \mathrm{keV}$ photon energy by using a higher-order diffraction for the analyzer, although a spectral range is limited to $4 \mathrm{eV}$ due to the small angular divergence of $2.7 \mathrm{mrad}$ [10]. In this study, we utilized a multi-layer mirror to achieve a large divergence of $22 \mathrm{mrad}$ to measure a wide spectral range of $55 \mathrm{eV}$, while keeping the resolution sufficient to resolve the spike structures of a few hundred meV.

\section{Design of Spectrometer}

The basic design of the dispersive X-ray spectrometer in this study consists of an elliptical mirror, a flat analyzer crystal, and a position sensitive detector [8]. First, the spectral resolution $d E / E$ is described by

$$
\frac{d E}{E}=\frac{\Psi}{\tan \theta_{B}} .
$$

where $E$ and $\theta_{\mathrm{B}}$ are the photon energy and the Bragg angle, respectively. $\Psi$ is determined by parameters of the X-ray source and optics $\Psi_{1}$ and the angular resolution of the detector $\Psi_{2}$, as given by

$$
\begin{gathered}
\Psi=\sqrt{\Psi_{1}^{2}+\Psi_{2}^{2}}, \\
\Psi_{1}=\frac{\sqrt{\sigma^{2}+L^{2} \omega^{2}}}{L},
\end{gathered}
$$

and

$$
\Psi_{2}=\frac{p}{L}
$$

Here, $\sigma, \omega, L$, and $p$ are the X-ray source size, the Darwin width of the Bragg reflection, the distance from the $\mathrm{X}$-ray source to the detector, and the pixel size of the detector, respectively.

Second, the spectral range $\Delta E$ is described by

$$
\frac{\Delta E}{E}=\frac{\Delta \theta}{\tan \theta_{\mathrm{B}}}
$$

Here, $\Delta \theta$ is the angular divergence of the $X$-ray beam on the analyzer crystal. Equation (5) shows that a larger divergence is required for expanding the covered spectral range. Although an elliptical mirror with total reflection was utilized in our previous experiment [10], the mirror cannot provide a sufficient divergence angle for measuring the entire spectrum in one shot due to its small reflection angle of a few mrad.

To overcome the limitation, we used platinum/carbon multi-layer mirrors [12], which were installed as the last optics of the two-stage focusing system at SACLA [13], to produce a large divergence $\Delta \theta$ of $22 \mathrm{mrad}$ with a central photon energy of $9070 \mathrm{eV}$ and a central incident angle of $14 \mathrm{mrad}$. We note that the reflectivity of the single mirror with 30 layers is $\sim 70 \%$ at a photon energy range between 8900 and $9300 \mathrm{eV}$. To obtain a high spectral resolution in the dispersive spectrometer, a large Bragg angle is required from Equation (1). For the distance $L$ of $1.5 \mathrm{~m}$ and a pixel size $p$ of $50 \mu \mathrm{m}$ for a Multi-Port Charge Coupled Device (MPCCD) detector [14] in the present geometry, we derive both the spectral range of $55 \mathrm{eV}$ and the resolution of $80 \mathrm{meV}$ at around $9070 \mathrm{eV}$, simultaneously, with a Bragg angle of $75.2^{\circ}$ using a silicon (553) analyzer crystal. 


\section{Experiment and Discussion}

Our experiment was conducted at BL3 of SACLA [15]. A schematic of the experimental setup is shown in Figure 1. XFEL pulses with a central photon energy of $9070 \mathrm{eV}$ were focused with the two-stage focusing system using platinum/carbon multi-layer mirrors. The silicon (553) analyzer crystal was set at $1.2 \mathrm{~m}$ from the focal point, and an MPCCD detector was placed on a 0.3-m-long detector arm. Thus, the total length from the focal point to the detector $L$ was $1.5 \mathrm{~m}$, as used in the estimation in Section 2. Figure 2a shows a typical single-shot spectrum of an XFEL pulse, demonstrating that the spectrometer is capable of covering the complete pulse spectrum. In Figure $2 b$, we show the enlarged spectrum around the peak maximum of Figure 2a. The typical width of observed spikes is $390 \pm 30 \mathrm{meV}$ in full width at half maximum (FWHM), which is sufficiently larger with respect to the resolution of the spectrometer of $80 \mathrm{meV}$. Based on this result, we estimated the XFEL pulse duration with a method described in [10]. Here, an energy chirp of the electron beam of $6 \times 10^{-5} \mathrm{fs}^{-1}$ was used as the simulation parameter. By comparing the measurement with a simulation, the pulse duration was estimated to be $7.7 \pm 1.1 \mathrm{fs}$ in FWHM. From the evaluation of Figure 2a, the longitudinal mode number was around 60 .

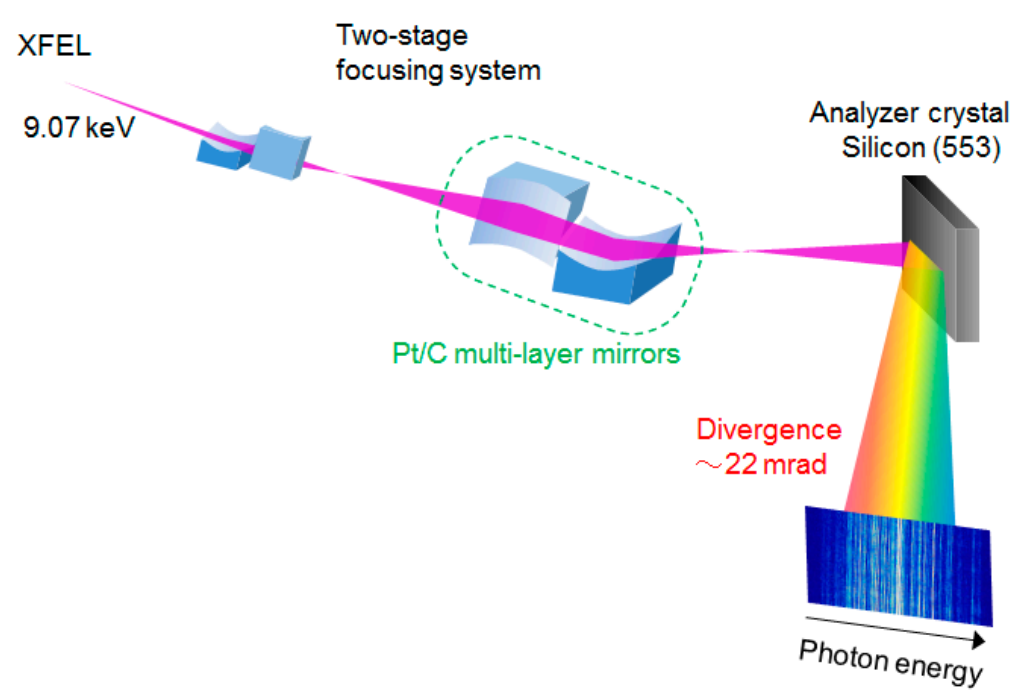

Figure 1. Experimental setup. The X-ray divergence was increased with the two-stage focusing optics, in which multi-layer elliptical mirrors were used as a second K-B optics. The spectral profile analyzed by a silicon (553) crystal is recorded with a MPCCD detector.

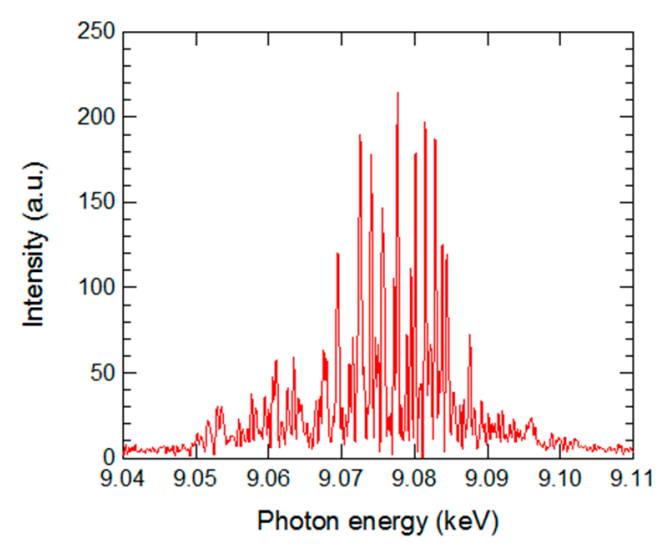

(a)

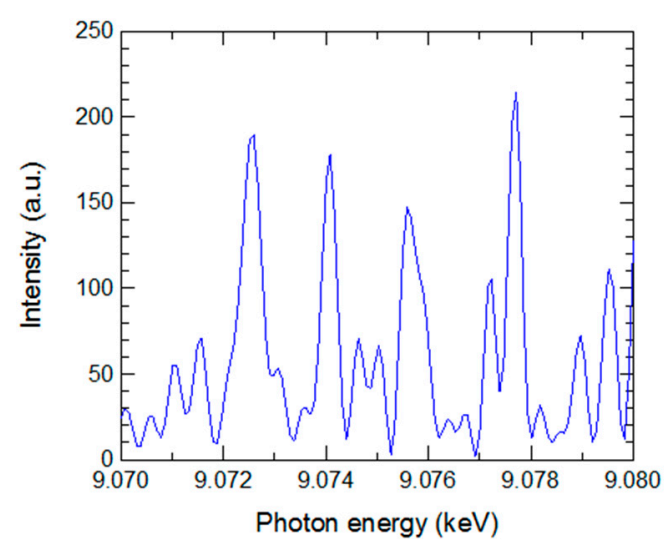

(b)

Figure 2. (a) Spectrum of the XFEL beam measured with the spectrometer; (b) enlarged spectrum around the central region of Figure $2 \mathrm{a}$. 


\section{Summary}

We demonstrated the measurement of the single-shot entire XFEL spectrum with fully resolved spike structures. In addition to the duration of the XFEL pulse, we obtained the longitudinal mode number. The results could provide new information to a theoretical study of XFEL physics. Moreover, the method is applicable to studies of X-ray nonlinear optics [5,16-18] because the mirrors in our spectrometer are a part of an XFEL two-stage focusing system. In particular, the quality of spectrum measurements for an absorption spectroscopy [18] and stimulated emissions [5] will be improved with a higher resolution over a wider energy range.

Acknowledgments: We would like to acknowledge the supporting members of SACLA facility. We also thank Takashi Tanaka and Ryota Kinjo for advice regarding spectral analysis. This research was partially supported by JSPS KAKENHI Grant Numbers 15H05434 and 23226004. The experiment was performed at BL3 of SACLA with the approval of the Japan Synchrotron Radiation Research Institute (JASRI) (proposal Nos. 2015A8009, $2015 \mathrm{~B} 8005$ and 2015B8013).

Author Contributions: Y.I. conceived and designed the experiment. J.K., A.N., S.M., H.Y., T.K., H.O. and K.Y. developed the X-ray optics. Y.I., I.I. and K.T. performed the experiment. Y.I. and M.Y. wrote the paper.

Conflicts of Interest: The authors declare no conflict of interest.

\section{References}

1. Emma, P.; Akre, R.; Arthur, J.; Bionta, R.; Bostedt, C.; Bozek, J.; Brachmann, A.; Bucksbaum, P.; Coffee, R.; Decker, F.-J.; et al. First lasing and operation of an ångstrom-wavelength free-electron laser. Nat. Photonics 2010, 4, 641-647. [CrossRef]

2. Ishikawa, T.; Aoyagi, H.; Asaka, T.; Asano, Y.; Azumi, N.; Bizen, T.; Ego, H.; Fukami, K.; Fukui, T.; Furukawa, Y.; et al. A compact X-ray free-electron laser emitting in the sub-ångstrom region. Nat. Photonics 2012, 6, 540-544. [CrossRef]

3. Chapman, H.N.; Fromme, P.; Barty, A.; White, T.A.; Kirian, R.A.; Aquila, A.; Hunter, M.S.; Schulz, J.; DePonte, D.P.; Weierstall, U.; et al. Femtosecond X-ray protein nanocrystallography. Nature 2011, 470, 73-77. [CrossRef] [PubMed]

4. Suga, M.; Akita, F.; Hirata, K.; Ueno, G.; Murakami, H.; Nakajima, Y; Shimizu, T.; Yamashita, K.; Yamamoto, M.; Ago, H.; et al. Native structure of photosystem II at $1.95 \AA$ A resolution viewed by femtosecond X-ray pulses. Nature 2015, 517, 99-103. [CrossRef] [PubMed]

5. Yoneda, H.; Inubushi, Y.; Nagamine, K.; Michine, Y.; Ohashi, H.; Yumoto, H.; Yamauchi, K.; Mimura, H.; Kitamura, H.; Katayama, T.; et al. Atomic inner-shell laser at 1.5-ångström wavelength pumped by an X-ray free-electron laser. Nature 2015, 524, 446-449. [CrossRef] [PubMed]

6. Bonifacio, R.; Pellegrini, C.; Narducci, L.M. Collective instabilities and high-gain regime in a free electron laser. Opt. Commun. 1984, 50, 373-378. [CrossRef]

7. Saldin, E.L.; Schneidmiller, E.A.; Shvyd'ko, Y.V.; Yurkov, M.V. X-ray FEL with a meV bandwidth. Nucl. Instrum. Methods Phys. 2001, 475, 357-362. [CrossRef]

8. Yabashi, M.; Hastings, J.B.; Zolotorev, M.S.; Mimura, H.; Yumoto, H.; Matsuyama, S.; Yamauchi, K.; Ishikawa, T. Single-Shot Spectrometry for X-ray Free-Electron Lasers. Phys. Rev. Lett. 2006, 97, 084802. [CrossRef] [PubMed]

9. Zhu, D.; Cammarata, M.; Feldkamp, J.M.; Fritz, D.M.; Hastings, J.B.; Lee, S.; Lemke, H.Y.; Robert, A.; Turner, J.L.; Feng, Y. A single-shot transmissive spectrometer for hard X-ray free electron lasers. Appl. Phys. Lett. 2012, 101, 034103. [CrossRef]

10. Inubushi, Y.; Tono, K.; Togashi, T.; Sato, T.; Hatsui, T.; Kameshima, T.; Togawa, K.; Hara, T.; Tanaka, T.; Tanaka, H.; et al. Determination of the Pulse Duration of an X-ray Free Electron Laser Using Highly Resolved Single-Shot Spectra. Phys. Rev. Lett. 2012, 109, 144801. [CrossRef] [PubMed]

11. Rich, D.; Zhu, D.; Turner, J.; Zhang, D.; Hill, B.; Feng, Y. The LCLS variable-energy hard X-ray single-shot spectrometer. J. Synchrotron Rad. 2015, 23, 3-9. [CrossRef] [PubMed]

12. Kim, J.; Nagahira, A.; Koyama, T.; Matsuyama, S.; Sano, Y.; Yabashi, M.; Ohashi, H.; Ishikawa, T.; Yamauchi, K. Damage threshold of platinum/carbon multilayers under hard X-ray free-electron laser irradiation. Opt. Express 2015, 23, 29032. [CrossRef] [PubMed] 
13. Mimura, H.; Yumoto, H.; Matsuyama, S.; Koyama, T.; Tono, K.; Inubushi, Y.; Togashi, T.; Sato, T.; Kim, J.; Fukui, R.; et al. Generation of $10^{20} \mathrm{~W} \mathrm{~cm}^{-2}$ hard X-ray laser pulses with two-stage reflective focusing system. Nat. Commun. 2014, 5, 3539. [CrossRef] [PubMed]

14. Kameshima, T.; Ono, S.; Kudo, T.; Ozaki, K.; Kirihara, Y.; Kobayashi, K.; Inubushi, Y.; Yabashi, M.; Horigome, T.; Holland, A.; et al. Development of an X-ray pixel detector with multi-port charge-coupled device for X-ray free-electron laser experiments. Rev. Sci. Instrum. 2014, 85, 033110. [CrossRef] [PubMed]

15. Tono, K.; Togashi, T.; Inubushi, Y.; Sato, T.; Katayama, T.; Ogawa, K.; Ohashi, H.; Kimura, H.; Takahashi, S.; Takeshita, K.; et al. Beamline, experimental stations and photon beam diagnostics for the hard X-ray free electron laser of SACLA. New J. Phys. 2013, 15, 083035. [CrossRef]

16. Tamasaku, K.; Nagasono, M.; Iwayama, H.; Shigemasa, E.; Inubushi, Y.; Tanaka, T.; Tono, K.; Togashi, T.; Sato, T.; Katayama, T.; et al. Double Core-hole Creation by Sequential Attosecond Photo-ionization. Phys. Rev. Lett. 2013, 111, 043001. [CrossRef] [PubMed]

17. Tamasaku, K.; Shigemasa, E.; Inubushi, Y.; Katayama, T.; Sawada, K.; Yumoto, H.; Ohashi, H.; Mimura, H.; Yabashi, M.; Yamauchi, K.; et al. X-ray two-photon absorption competing against single and sequential multiphoton processes. Nat. Photonics 2014, 8, 313. [CrossRef]

18. Yoneda, H.; Inubushi, Y.; Yabashi, M.; Katayama, T.; Ishikawa, T.; Ohashi, H.; Yumoto, H.; Yamauchi, K.; Mimura, H.; Kitamura, H. Saturable absorption of intense hard X-rays in iron. Nat. Commun. 2014, 5, 5080. [CrossRef] [PubMed]

(C) 2017 by the authors. Licensee MDPI, Basel, Switzerland. This article is an open access article distributed under the terms and conditions of the Creative Commons Attribution (CC BY) license (http:/ / creativecommons.org/licenses/by/4.0/). 